\title{
Branch Retinal Vein Occlusion after Messenger RNA-Based COVID-19 Vaccine
}

\author{
Kazunobu Sugihara Michihiro Kono Masaki Tanito \\ Department of Ophthalmology, Shimane University Faculty of Medicine, Izumo, Japan
}

\section{Keywords}

Branch retinal vein occlusion - Coronavirus disease 2019 - BNT162b2 - Messenger RNA ·

Thrombosis · Eye

\begin{abstract}
Two days after the second dose of the messenger RNA-based COVID-19 vaccine (BNT162b2), a healthy 38-year-old man developed branch retinal vein occlusion (BRVO) in his left eye (OS). His previous medical history was unremarkable and he was a nonsmoker. His blood pressure was $117 / 78 \mathrm{~mm} \mathrm{Hg}$. Blood examination did not suggest thrombophilia. His best-corrected visual acuity (BCVA) was 0.9 OS with myopic correction. A fundus examination showed a retinal hemorrhage and cotton wool spots in the superotemporal region of the posterior pole OS. Optical coherence tomography macular scans showed subfoveal fluid accumulation and retinal thickening in the superior macular region OS. Two intravitreal injections of aflibercept were administered 2 months apart. By 7 months after the initial visit, the BCVA was 1.2 OS and the retinal hemorrhage and macular edema have resolved. BRVO can be seen after BNT162b2 vaccinations. Because the third doses of the vaccine are beginning to be administered more widely, ocular complications including RVO can develop and require attention.
\end{abstract}

\section{Introduction}

COVID-19 became a pandemic in March 2020. Vaccines, the most efficient means to control this pandemic, are now being administered, worldwide. The messenger RNA-based COVID-19 vaccine (BNT162b2; Pfizer-BioNTech, New York, NY, USA and Mainz, Germany) is 
reported to be less likely to cause thrombotic adverse events compared with the adenovirus vector COVID-19 vaccine (ChAdOx1; Oxford-AstraZeneca, Cambridge, UK) [1, 2]. However, although rare, a case of central retinal vein occlusion (CRVO) after the second dose of the BNT162b2 vaccine was reported, recently [3]. Here, we report a case of branch retinal vein occlusion (BRVO) after a second dose of the same vaccine.

\section{Case Report}

A healthy 38-year-old man was referred to our department for decreased vision in his left eye (OS) 15 days after the second dose of the BNT162b2 vaccine. His previous medical history was unremarkable, and he was a nonsmoker. After the first vaccine dose, he had a mild flu-like malaise for a day. After the second dose, he had a flu-like malaise with a fever of $37.8^{\circ} \mathrm{C}$. Two days after the vaccination, he reported difficulty seeing the computer monitor. Because of further visual deterioration 11 days after the vaccination, he visited his local ophthalmologist 15 days after the vaccination who diagnosed a retinal hemorrhage OS. He was referred to our department on the same day.

At the initial visit to our department, his best-corrected visual acuity (BCVA) was 1.2 in his right eye and 0.9 OS with myopic correction. A fundus examination showed a retinal hemorrhage and cotton wool spots in the superotemporal region of the posterior pole OS (Fig. 1a); no pathological findings were observed in the right eye. Optical coherence tomography macular scans showed subfoveal fluid accumulation and retinal thickening (central foveal thickness = $373 \mu \mathrm{m}$ ) in the superior macular region OS (Fig. 1b,c). Fluorescein angiography showed vascular staining and mild macular leakage OS (Fig. 1d, e). His blood pressure was 117/78 mm Hg. Blood examination including platelet count $\left(256 \times 10^{3} / \mu \mathrm{L}\right)$, D-dimer $(<0.5 \mu \mathrm{g} / \mathrm{mL})$, protein-C $(147 \%)$, protein-S (124\%), anticardiolipin-beta2-glycoprotein I complex antibody $(<0.7 \mathrm{U} / \mathrm{mL})$, and anticardiolipin immunoglobulin $\mathrm{G}(8.1 \mathrm{U} / \mathrm{mL})$ were not deviated remarkably from the normal range; thrombophilia was not suspected. He was diagnosed with BRVO and macular edema OS.

At the initial visit, an intravitreal injection of vascular endothelial growth factor inhibitor (2 mg/0.05 mL aflibercept, Eylea; Santen Pharmaceutical, Osaka, Japan) was administered. Three weeks after the injection, the BCVA was $0.8 \mathrm{OS}$, and the macular edema and serous retinal detachment improved (Fig. 2a, b). Two months after the injection, the BCVA was 1.2 OS; because intraretinal fluid OS was still seen on the optical coherence tomography scans, a second aflibercept ( $2 \mathrm{mg} / 0.05 \mathrm{~mL}$ ) injection was administered. By 7 months after the initial visit, the BCVA was 1.2 OS and the retinal hemorrhage and macular edema have resolved (central foveal thickness $=272 \mu \mathrm{m})($ Fig. 3a, b).

\section{Discussion and Conclusion}

Current reports have suggested that the frequencies of thrombocytopenia and thromboembolic and hemorrhagic events after vaccination are increasing with the ChAdOx1 vaccine but might not be increasing after the BNT162b2 vaccine in the general population $[1,2]$. A case of bilateral ocular venous thrombosis, simple thrombocytopenia, and ischemic brain stroke after the ChAdOx1 vaccine have been reported as ocular adverse events [4]. Regarding the BNT162b2 vaccine, a single case of CRVO developed immediately after the second dose of the vaccine [3]. The current case of development of BRVO after the BNT162b2 vaccine is unique in the literature.

The current patient had no remarkable background risks for thrombocytopenia and venous thrombus before and after the first vaccine dose. In the previous case, CRVO developed 

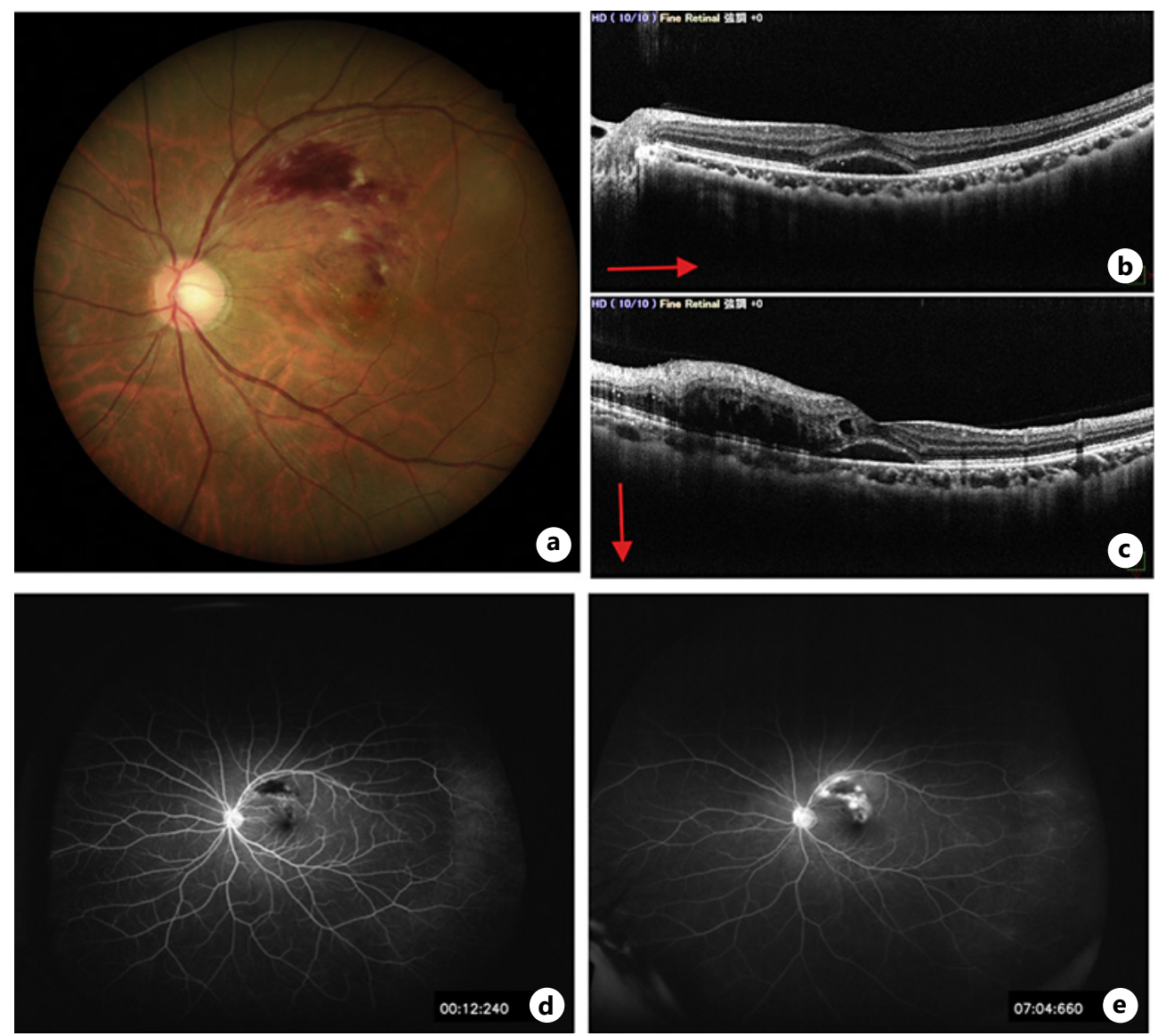

Fig. 1. A fundus photography (a), horizontal- (b) and vertical-OCT (c), and FA findings OS at the initial visit (d, e). a A retinal hemorrhage and cotton wool spots are seen in the flow region of the superotemporal vein OS. b, c OCT scans suggest the presence of intra- and subretinal fluid accumulation. The red arrows indicate the OCT scan direction. Early- (d) and late-phase (e) FA scans suggest fluorescein pooling but no neovascularization. OCT, optical coherence tomography; FA, fluorescein angiography.

after the second dose in a healthy 50 -year-old nonobese man who was a nonsmoker and had no remarkable serologic abnormalities [3]. Given this report of a single case of BRVO, the underlying mechanism of RVO remains unclear; however, different levels/pathways of immune responses between the first and second doses might be involved in the development of retinal venous thrombosis.

Patients who had no problems with two vaccine doses but developed vaccine-induced thrombotic thrombocytopenia after the third (booster) dose [5] were reported. As the third doses of the vaccines are being administered widely, ocular complications including CRVO and BRVO may develop and require attention.

\section{Statement of Ethics}

This study adhered to the tenets of the Declaration of Helsinki. This retrospective review of patient data did not require ethical approval in accordance with the Ethical Guidelines for Medical and Health Research Involving Human Subjects in Japan. The patient provided written informed consent for publication of this case report and any accompanying pictures. 


\section{Case Reports in Ophthalmology}

\begin{tabular}{l|l}
\hline Case Rep Ophthalmol 2022;13:28-32 \\
\hline DOI: 10.1159/000521838 & $\begin{array}{l}\text { @ 2022 The Author(s). Published by S. Karger AG, Basel } \\
\text { www.karger.com/cop }\end{array}$ \\
\hline
\end{tabular}

Sugihara et al.: BRVO after COVID-19 Vaccine

Fig. 2. Horizontal- (a) and vertical-mOCT (b) findings OS 3 weeks after the intravitreal aflibercept injection. The macular edema has improved. The red arrows indicate the OCT scan direction. OCT, optical coherence tomography.
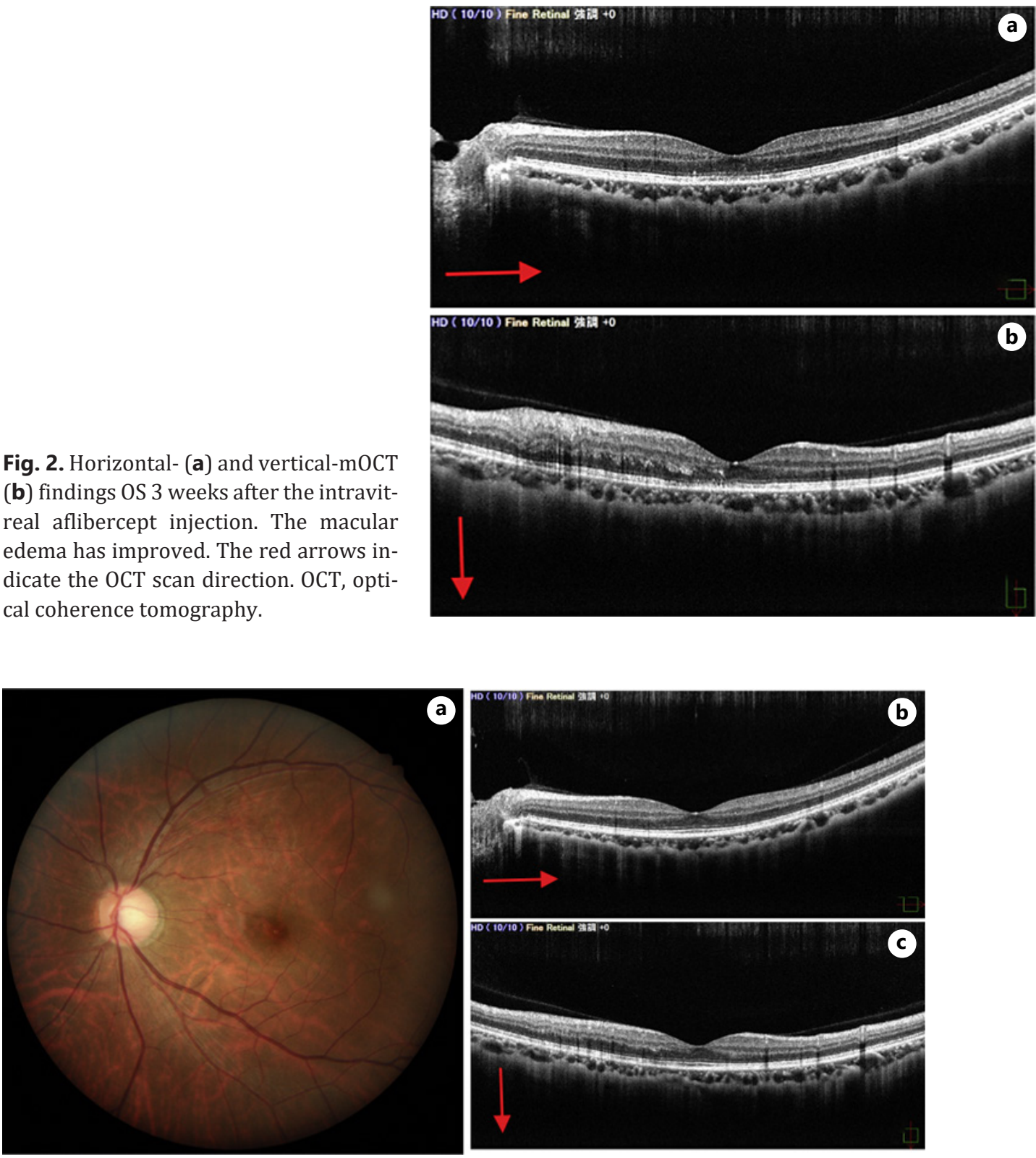

Fig. 3. Fundus photograph (a) and horizontal- (b) and vertical-OCT (c) findings OS 7 months after the initial visit. The retinal hemorrhage (a) and macular edema have resolved (b, c). The red arrows indicate the OCT scan direction. OCT, optical coherence tomography.

\section{Conflict of Interest Statement}

The authors have no conflicts of interest associated with this report.

\section{Funding Sources}

No financial support was provided.<smiles></smiles> 
Sugihara et al.: BRVO after COVID-19 Vaccine

\section{Author Contributions}

K.S. and M.K. treated the subject and collected the clinical data. K.S. and M.T. wrote the manuscript, and M.K. revised the manuscript. All authors approved the final version of the manuscript. The authors agree to be responsible for all aspects of this work.

\section{Data Availability Statement}

All data generated or analyzed during this study are included in this article. Further inquiries can be directed to the corresponding author.

\section{References}

1 Cari L, Fiore P, Naghavi Alhosseini M, Sava G, Nocentini G. Blood clots and bleeding events following BNT162b2 and ChAdOx1 nCoV-19 vaccine: an analysis of European data. J Autoimmun. 2021;122:102685.

2 Simpson CR, Shi T, Vasileiou E, Katikireddi SV, Kerr S, Moore E, et al. First-dose ChAdOx1 and BNT162b2 COVID-19 vaccines and thrombocytopenic, thromboembolic and hemorrhagic events in Scotland. Nat Med. 2021;27(7):1290-7.

3 Bialasiewicz AA, Farah-Diab MS, Mebarki HT. Central retinal vein occlusion occurring immediately after 2nd dose of mRNA SARS-CoV-2 vaccine. Int Ophthalmol. 2021;41(12):3889-92.

4 Bayas A, Menacher M, Christ M, Behrens L, Rank A, Naumann M. Bilateral superior ophthalmic vein thrombosis, ischaemic stroke, and immune thrombocytopenia after ChAdOx1 nCoV-19 vaccination. Lancet. 2021; 397(10285):e11.

5 Malayala SV, Papudesi BN, Sharma R, Vusqa UT, Raza A. A case of idiopathic thrombocytopenic purpura after booster dose of BNT162b2 (Pfizer-Biontech) COVID-19 vaccine. Cureus. 2021;13(10):e18985.

\section{Karger's}

